\title{
Long-term survival after traumatic brain injury: A population-based analysis
}

\author{
Allen W. Brown ${ }^{\mathrm{a}, *}$, Cynthia L. Leibson ${ }^{\mathrm{b}}$, James F. Malec ${ }^{\mathrm{c}}$, Patricia K. Perkins ${ }^{\mathrm{b}}$, Nancy N. Diehl ${ }^{\mathrm{b}}$ and \\ Dirk R. Larson ${ }^{\mathrm{b}}$ \\ ${ }^{a}$ Department of Physical Medicine and Rehabilitation, Mayo Clinic, Rochester, MN, USA \\ ${ }^{\mathrm{b}}$ Department of Health Sciences Research, Mayo Clinic, Rochester, MN, USA \\ ${ }^{\mathrm{c}}$ Department of Psychiatry and Psychology, Mayo Clinic, Rochester, MN, USA
}

\begin{abstract}
This population-based retrospective cohort study identified all Olmsted County, MN residents with any diagnosis indicative of potential traumatic brain injury (TBI) during the years 1985 to 2000 . The complete community-based medical records of a random sample ( $n=7,175$ ) were reviewed to confirm and characterize the event, and to determine vital status through 2002. The review identified 1,448 confirmed incident cases; 164 (11\%) were moderate to severe; 1,284 were mild. The estimated 30-day case fatality rate was $29 \%$ for moderate to severe cases and $0.2 \%$ for mild cases. Comparison of observed mortality over the full period of follow-up with that expected revealed a risk ratio (95\% CI) of 5.29 (4.11-6.71) for moderate to severe cases and 1.33 (1.05-1.65) for mild cases. Proportional hazards modeling showed the adjusted hazard of all-cause mortality for moderate to severe cases relative to mild cases was 5.18 (3.65-7.3) within six months of the event and 1.04 (0.57-1.88) for the remaining follow-up period. This analysis indicates that persons who experience mild TBI exhibit a small but statistically significant reduction in long-term survival compared to the general population. The case fatality rate for persons with moderate to severe TBI is very high, but among six-month survivors, long-term survival is similar to that for persons with mild TBI.
\end{abstract}

Keywords: Brain injuries, head trauma, longevity, mortality

\section{Introduction}

Traumatic brain injury (TBI) can result in lasting neurological impairment, including cognitive dysfunction and psychosocial disturbance, that may increase risk for secondary medical morbidity and mortality. The aging of the United States population, as well as improvement in acute medical and surgical care, will increase the potential incidence and associated disability of non-fatal TBI. These factors emphasize the importance of understanding the relationship between TBI, injury severity, and long-term survival to help in the development of clinical and community-based programs to provide services for this growing population.

*Address for correspondence: Allen W. Brown, MD, Department of Physical Medicine and Rehabilitation, Mayo Clinic, Rochester, MN 55905, USA. Tel.: +1 507255 3116; Fax: +1 507255 7696; E-mail: brown.allen@mayo.edu.
Though TBI is well recognized to be associated with significant mortality in the acute period $[7,9,11$, 20,22] survival after the acute period is less well defined. Studies are few and mostly from restricted populations. An early report compared life expectancy in male World War I veterans who experienced primarily severe war-related head injuries, with and without subsequent epilepsy, with that for a population of noninjured veterans [23]. The study concluded that life expectancy of head-injured men was reduced by 3 to 5 years compared to age-matched controls, with the reduction at the maximum after age 50 and associated with post-traumatic epilepsy. Further study of the same population confirmed the association of post-traumatic epilepsy with reduced life expectancy [24]. A more recent study of male American military personnel, who sustained a penetrating craniocerebral injury during the Vietnam War and survived for a minimum of one week, showed that the population appeared to be approach- 
ing actuarial norms of life expectancy 15 years after injury [14]. The study was limited to persons who required neurosurgical procedures, and the authors found no association between post-traumatic epilepsy and risk of death. Coma on presentation or the persistence of coma was most predictive of death. An analysis of a clinical case series of survivors of TBI who were admitted to a major teaching hospital in Australia for inpatient rehabilitation [6] found a significantly increased mortality rate among persons with TBI compared to the expected rate for an equivalent population.

The California Department of Developmental Services data base has been extensively analyzed, studying life expectancy and cause of death in adults and children with disabling neurological impairment $[15$, 17-19]. This database includes assessments of psychological, medical, behavioral and cognitive function. In a study of long-term causes of death after TBI in persons aged 10 or older who survived greater than 12 months, long-term mortality was elevated compared to the general population [15]. Mortality was greatest during the subsequent 4-year period and was associated with reduced mobility.

Analysis of long-term survival after TBI for nonrestricted populations in the United States is lacking. The objectives of the analysis reported here are: (1) to compare observed versus expected survival following TBI for all members of the Olmsted County Minnesota $(\mathrm{MN})$ population who experienced any traumatic brain injuries that were medically attended between 1985 and 2000 , stratified by injury severity, and (2) to compare survival for moderate to severe cases with that of mild cases, adjusted for age, gender, and calendar year of the event.

\section{Methods}

\subsection{Study setting}

Olmsted County, Minnesota, (2000 census population, 124,277) provides a unique opportunity for investigating the natural history of TBI [2-5,13]. Rochester, the county seat, is approximately 80 miles from the nearest major metropolitan area and is home to one of the world's largest private medical centers, the Mayo Clinic. The Mayo Clinic and its two affiliated hospitals or the Olmsted Medical Center, a second group practice, and its affiliated hospital provide nearly all of the medical care delivered to local residents. Since 1907, every Mayo Clinic patient has been assigned a unique identifier, and all information from every contact (including office, emergency department, and nursing home visits and hospital inpatient or outpatient admissions) is contained within a single dossier for each patient. The detailed information includes a medical history, clinical assessments, consultation reports, surgical procedures, dismissal summaries, laboratory and radiology results, correspondence, and death certificates. The diagnoses assigned at each visit are coded and entered into continuously updated computer files. These files constitute the Rochester Epidemiology Project (REP) diagnostic index [12]. This index was expanded to include the few other providers of medical care to local residents, including the Olmsted Medical Center and the few private practitioners in the area, thereby linking the medical records for community residents. The REP provides the capability for population-based studies of disease risk factors and outcomes that is unique in the United States.

\subsection{Study population}

This study was approved by the Mayo Clinic and the Olmsted Medical Center Institutional Review Boards. All Olmsted County residents with any diagnosis suggestive of traumatic brain injury (TBI) in the REP diagnostic index from 1/1/1985 through 12/31/1999 were identified as part of the Mayo Traumatic Brain Injury Model System research activities funded by the National Institute on Disability and Rehabilitation Research (NIDRR). A 15.7\% random sample was selected for review of their complete community-based medical records from date first seen until date last seen at any REP provider. Trained nurse abstractors under the direction of a board-certified physiatrist $(\mathrm{AB})$ and neuropsychologist (JM) performed the review.

For purposes of this study, TBI was defined as a traumatically induced injury that contributed to physiological disruption of brain function. Evidence of physiological disruption included documentation of any of the following: concussion with loss of consciousness, traumatic amnesia (either retrograde or anterograde), neurological signs of brain injury and/or evidence of intracerebral, subdural, or epidural hematoma, cerebral or hemorrhagic contusion, or brain stem injury; penetrating brain injury; skull fracture; or post-concussive symptoms (dizziness, confusion, blurred vision, double vision, headache, nausea, or vomiting that lasted greater than 30 minutes and that was not attributable to pre-existing or co-morbid conditions). Individuals for whom their clinical diagnosis was based on history 
alone (i.e., who did not present for medical care for either the event or for sequelae) were excluded as cases.

All incident TBI events were further characterized by severity (moderate to severe vs. mild) using all available clinical data including radiological imaging findings, surgical records, and autopsy protocol reviews. Events that included one or more of the following were categorized as moderate to severe: skull fracture, intracranial hematoma, brain contusion, penetrating skull injury, brain stem injury, or severe complications of TBI (e.g., neurological surgery, central nervous system infection, subarachnoid hemorrhage, hydrocephaly, leakage of cerebrospinal fluid). Events were categorized as mild if they were excluded from the moderate to severe group and had one or more of the following: loss of consciousness, amnesia, post-concussive symptoms, or focal neurological signs of brain injury.

Vital status and date of death for those who died was determined by review of the medical records and State of Minnesota death tapes. Persons for whom vital status as of the present was not known were censored as of the date they were last known to be Olmsted County residents.

\subsection{Analysis}

Both 30 day and long-term survival were analyzed for moderate to severe and mild cases separately by means of the Kaplan-Meier method. Long-term survival was also assessed for six-month survivors. Observed survival was compared with the age- and sexspecific expected survival of the 1990 Minnesota white population using the log-rank statistic. Multivariate proportional-hazards modeling of survival free of death was performed to test for differences between moderate to severe TBI and mild TBI adjusted for the effects of age, sex, and calendar year of the event. Tests for first-order interactions were examined, together with tests for the assumptions of proportionality.

\section{Results}

There were 45,831 residents of Olmsted County, $\mathrm{MN}$, with one or more diagnoses potentially indicative of TBI in the REP diagnostic index from 1/1/1985 through 12/31/1999. Review of the $15.7 \%(n=7,175)$ random sample yielded 1,448 who met criteria for a confirmed TBI incident case; 164 (11\%) were moderate to severe (mean \pm standard deviation age $=35.3$ \pm 30.5 years, $61 \%$ male) and 1,284 (89\%) were mild (mean age $=26.8 \pm 20.7$ years, $55 \%$ male). The mean duration of follow-up was 7.4 years (median $=6.8$ years; range $=0-18.2$ years).

Among moderate to severe cases, there were 68 deaths during 888 person-years of follow-up. The Kaplan-Meier estimated 30-day case fatality rate was $29.3 \%$ (95\% CI $=22.0-35.9)$; $20.1 \%$ of moderate to severe cases died on the day of the event. The 68 deaths over the full period of follow-up were significantly more than the 12.8 expected $(\mathrm{RR}=5.29,95 \%$ $\mathrm{CI}=4.11-6.71, p<0.001)$ (Fig. 1). However, among moderate to severe cases who survived six months, the observed 14 deaths did not differ significantly from the 12.7 expected $(\mathrm{RR}=1.10,95 \% \mathrm{CI}=0.60-1.85, p=$ 0.72) (Fig. 2).

Among mild cases, there were 78 deaths during 9,800 person-years of follow-up. The Kaplan-Meier estimated 30-day case fatality rate was $0.2 \%(95 \% \mathrm{CI}=$ 0.0-0.4). The 78 deaths over the full period of followup were significantly greater than the 58.8 expected $(\mathrm{RR}=1.33,95 \% \mathrm{CI}=1.05-1.65, p=0.012)($ Fig. 3). Among mild cases who survived six months, the observed 69 deaths were not significantly different than the 58.6 expected $(\mathrm{RR}=1.18,95 \% \mathrm{CI}=0.92-1.49$, $p=0.173$ ) (Fig. 4).

For all cases combined, multivariable Cox proportional-hazards modeling was performed to test for a significant difference between moderate to severe and mild cases, adjusted for age, sex, and calendar year. Tests revealed that the assumption of proportionality of hazards was violated. Thus, based on the Kaplan-Meier survival curves, we elected to investigate survival from event date to six months and after six months. Survival within six months of the event for persons with moderate to severe TBI was markedly reduced compared to those with mild TBI $(\mathrm{RH}=5.18,95 \% \mathrm{CI}=3.65-$ 7.30; $p<0.001)$. The significant interaction revealed that the relative hazard varied as a function of age, i.e., the higher six-month mortality for moderate to severe vs. mild TBI was greater at younger ages (Table 1a). Among individuals who survived six months from the event, the long-term survival for persons who experienced moderate to severe TBI did not differ from that for persons who experienced mild TBI $(\mathrm{RH}=1.04$; 95\% CI $=0.57-1.88, p=0.91$ ) (Table $1 \mathrm{~b})$.

\section{Discussion}

This report provides a unique population-based analysis of long-term survival following TBI in a United 


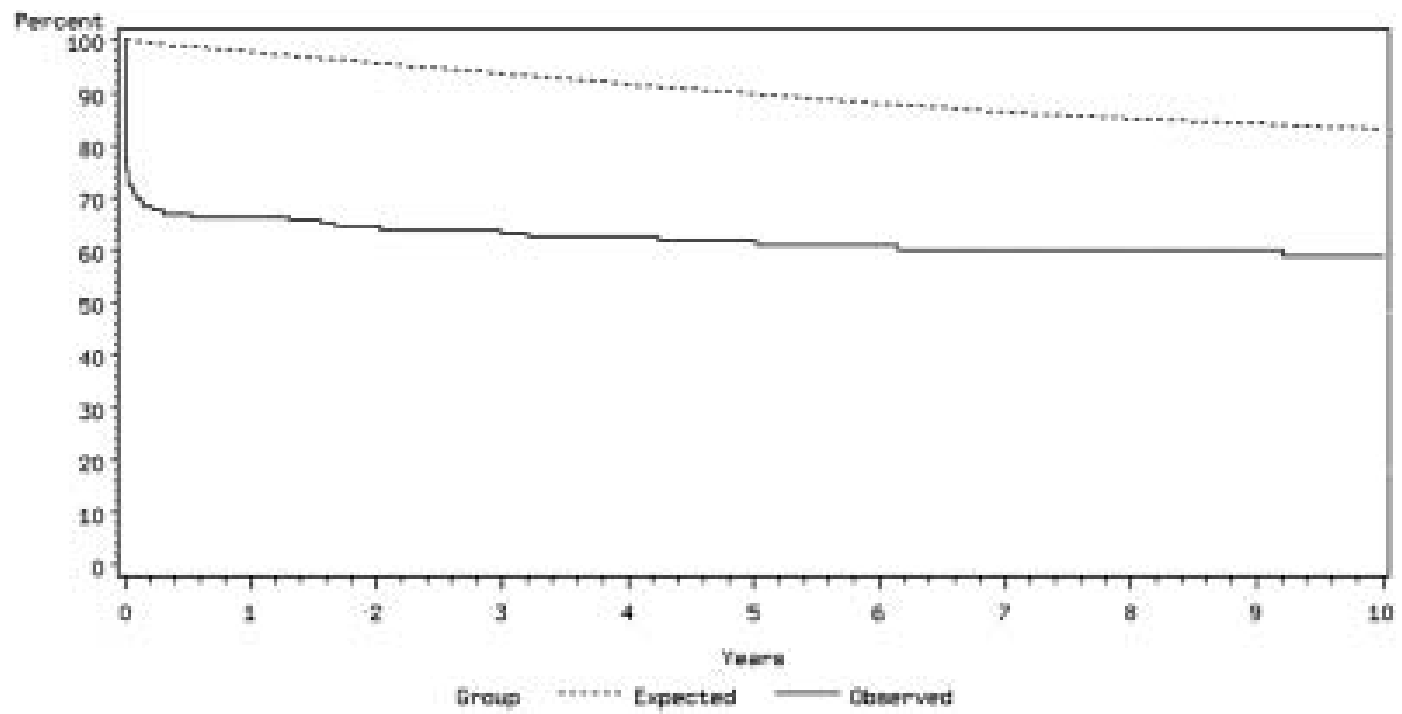

Fig. 1. Kaplan-Meier curves of observed and expected 10-year survival for 164 residents of Olmsted County, MN, with moderate to severe traumatic brain injury 1985-2000. Expected survival was calculated from mortality for the Minnesota white population of similar age, sex, and calendar year distribution.

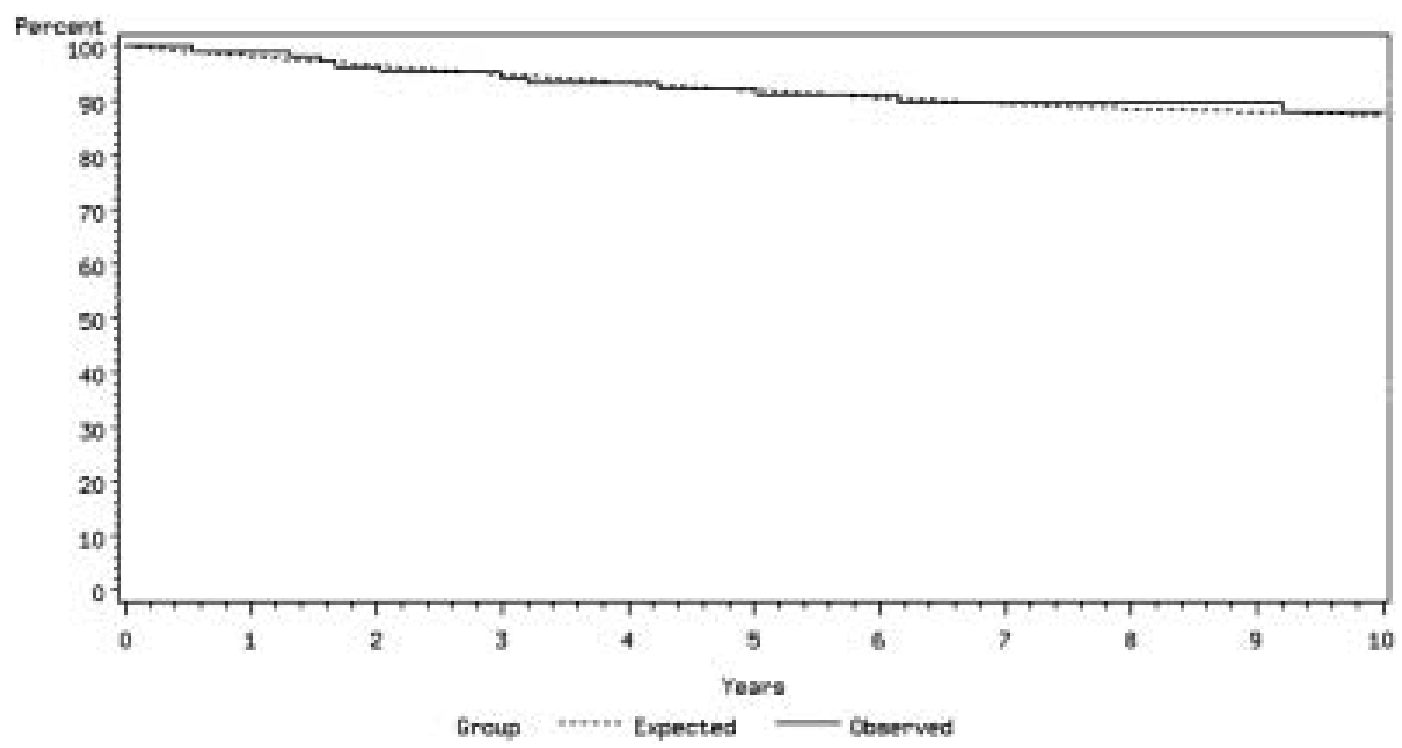

Fig. 2. Kaplan-Meier curves of observed and expected 10-year survival for moderate to severe cases who survived six months from the event. Expected survival was calculated from mortality for the Minnesota white population of similar age, sex, and calendar year distribution.

States population stratified by injury severity. It confirms the markedly reduced acute survival after moderate to severe injuries $[1,7,8,20]$. The most striking findings in this analysis are the modest but significant reduction in long-term survival after mild TBI and the lack of reduction in long-term survival among persons with moderate to severe injuries who survived six months. Given the high incidence of mild TBI, even a modest reduction in life expectancy is likely to have a significant social and economic effect [10]. In this analysis, although the percentage of people with mild TBI who died is small compared to the percentage that died following moderate to severe TBI, the actual number of those who died after mild injury (78) is greater than the number who died from moderate to severe injury (68). The significant negative effect of mild TBI 


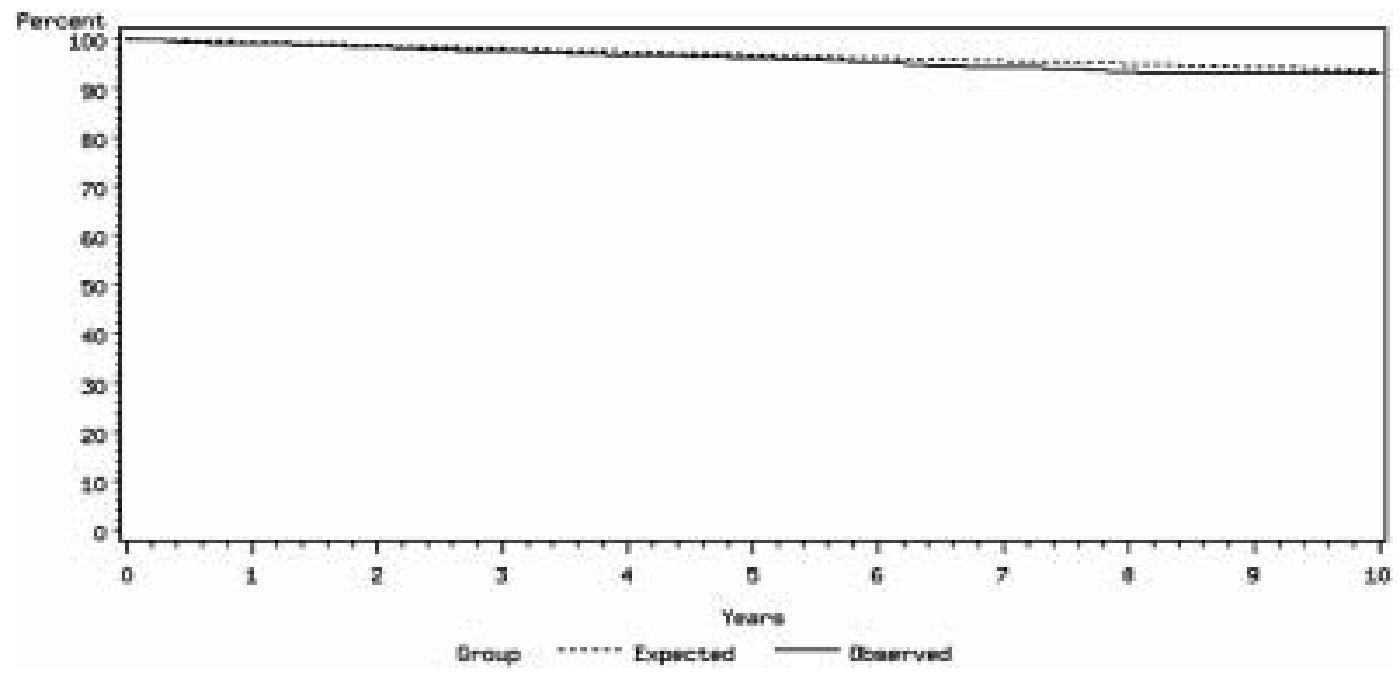

Fig. 3. Kaplan-Meier curves of observed and expected 10-year survival for 1,284 residents of Olmsted County, MN, with mild traumatic brain injury 1985-2000. Expected survival was calculated from mortality for the Minnesota white population of similar age, sex, and calendar year distribution.

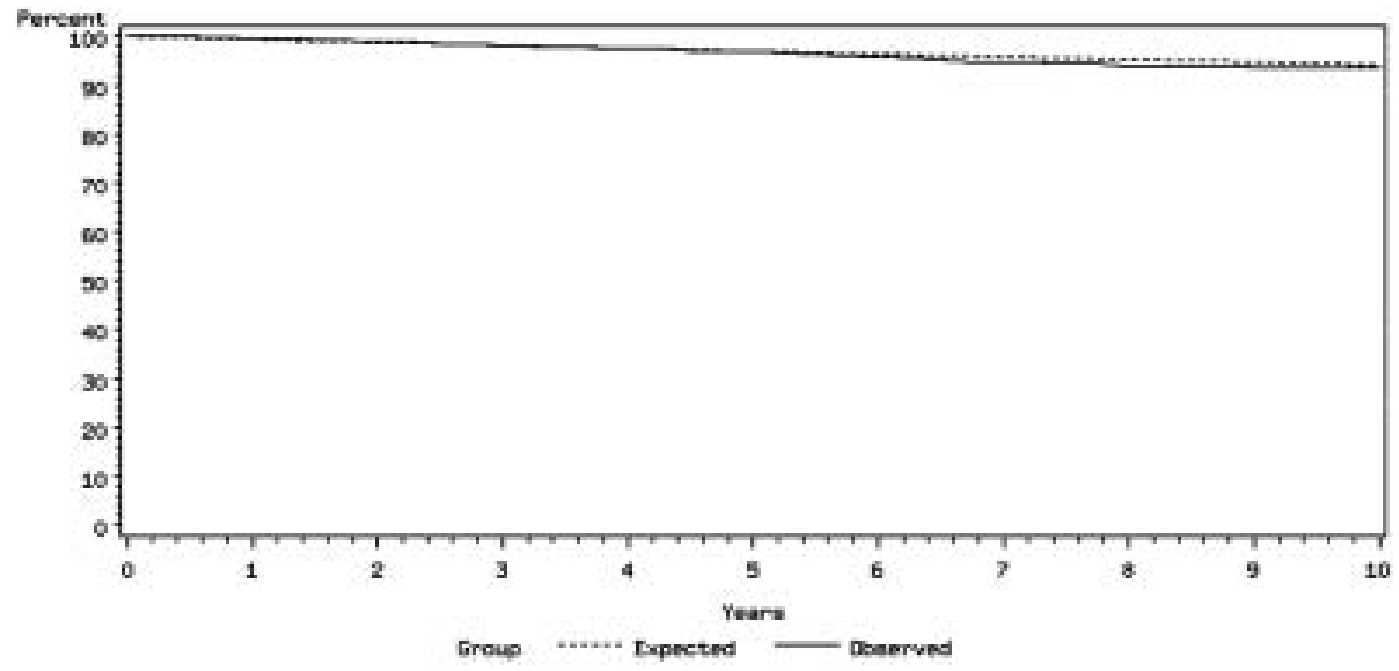

Fig. 4. Kaplan-Meier curves of observed and expected 10-year survival for mild cases who survived six months from the event. Expected survival was calculated from mortality for the Minnesota white population of similar age, sex, and calendar year distribution.

on long-term survival was no longer apparent when limited to persons who survived mild TBI for greater than six months. The extent to which the six-month mortality was directly related to head trauma cannot be determined from this analysis. However, our finding that persons with mild TBI experienced increased mortality from any cause deserves attention given the dramatic declines in hospitalization rates for persons with mild TBI [21]. The lack of reduction in long-term survival among persons who survived six months after moderate to severe injury when compared to mild injury is surprising and contrary to many previous reports $[6,14,15,23,24]$. If this finding can be confirmed in other populations, it would have implications for outcome prognostication, counseling, and community service development for people surviving TBI of all ages.

Recent trends indicating an increase incidence of fall-related TBI [8], with fall-related death rates now surpassing motor-vehicle-related TBI death rates in the United States among the elderly [1], have significant implications for aging Americans. As the population 
Table 1

Results of Cox proportional hazards modeling of survival free of death among persons with moderate to severe traumatic brain injury (TBI) compared to those with mild TBI

\begin{tabular}{|c|c|c|c|}
\hline Variable & Hazard Ratio & $95 \% \mathrm{CI}$ & P Value \\
\hline \multicolumn{4}{|c|}{ a. Modeled from time of event to six months } \\
\hline Age & - & - & $<0.001$ \\
\hline Male sex & 1.25 & $0.89-1.75$ & 0.195 \\
\hline Calendar year & 0.98 & $0.94-1.02$ & 0.357 \\
\hline Severe vs. mild & - & - & $<0.001$ \\
\hline Severity X Age & - & - & $<0.001$ \\
\hline \multicolumn{4}{|c|}{ b. Modeled from six months after event to date of last follow-up } \\
\hline Age & 1.08 & $1.07-1.10$ & $<0.001$ \\
\hline Male sex & 1.19 & $0.76-1.86$ & 0.443 \\
\hline Calendar year & 1.03 & $0.97-1.10$ & 0.325 \\
\hline Severe vs. mild & 1.04 & $0.57-1.88$ & 0.908 \\
\hline
\end{tabular}

ages, increasing risk for fall-related TBI, and as advances in emergency medical and surgical treatment further increase survival, the number of elderly persons acquiring new or additional disabling impairment in this country will increase. Additional age-related comorbidity is likely to influence outcome such as independent living. People who survive TBI may anticipate a normal life span but may also be at increased risk for subsequent TBI [4]. As survivors of TBI age with disability and an increased risk of additional injury, communities will need to plan for increasing utilization of health care and other supportive resources.

\section{Study limitations}

Data for this study were collected from a single population, which in 1990 was $96 \%$ white. With the exception of a higher proportion of the working population employed in the health care industry, the characteristics of Olmsted County are similar to those of United States whites. However, the under-representation of minorities in this cohort, and the fact that essentially all care was delivered by two institutions, compromises the generalizability to different racial and ethnic groups and to different health care environments. Because no single county or health care system can be expected to be completely representative of the general population, our findings should be further examined in different geographic and care settings. The relatively high standard of living and ready access to health care in this community may have led to improved survival and an over estimation relative to other communities. If so, the finding of reduced survival after mild TBI in this population gains significance.

The criteria used to categorize mild and moderate to severe injuries in this analysis were based on cri- teria used in previous work in this population [2-5]. Using these criteria, it is possible that some mild injuries were included in the moderate to severe group, such as those with skull fracture only. On the other hand, the definition of mild injury restricted this group to those with no structural evidence of injury. Other potential indicators of injury severity such as Glasgow Coma Scale (GCS), duration of post-traumatic amnesia (PTA), and duration of loss of consciousness are variably documented in the record. Reliance on these elements for case identification would have significantly restricted the sample. For this initial study, we elected to use criteria that allowed us to examine the largest portion of the population-based sample. Future analyses will examine the potential effect on survival rates of varying models for stratifying injury severity based on available data such as GCS and PTA. It has been reported that only $85 \%$ of persons experiencing mild and moderate head injuries seek medical attention [16]. Although the likelihood that TBI events are undetected is arguably lower in this setting relative to others, given the longitudinal and comprehensive nature of the REP medical records linkage system, we recognize that not all persons with mild injury come to medical attention.

\section{Conclusions}

This population-based analysis indicates that persons who experience mild TBI exhibit a small but statistically significant reduction in long-term survival compared to the general population. The case fatality rate for persons with moderate to severe TBI is very high, but among six-month survivors, long-term survival is similar to that for persons with mild TBI. The increased mortality among survivors of mild injury may have significant social and economic effects given the high incidence of mild TBI. The lack of difference among sixmonth survivors of moderate to severe and mild injuries may increase the need for supportive community services for individuals ageing with TBI-related disability at all levels of injury severity. These findings must be confirmed in different geographic and care settings to determine whether they can be generalized to other populations.

\section{Acknowledgments}

The authors gratefully acknowledge the excellent contribution of Margaret Donohue, RN for medical 
record abstraction. This study was supported by a TBI Model System grant from the National Institute on Disability and Rehabilitation Research (NIDRR), US Department of Education.

\section{References}

[1] N. Adekoya, D.J. Thurman, D.D. White and K.W. Webb, Surveillance for traumatic brain injury deaths - United States, 1989-1998, Morbidity \& Mortality Weekly Report, Surveillance Summaries 51 (2002), 1-14.

[2] J.F. Annegers and S.P. Coan, The risks of epilepsy after traumatic brain injury, Seizure 9 (2000), 453-457.

[3] J.F. Annegers, J.D. Grabow, R.V. Groover, E.R. Laws, Jr., L.R. Elveback and L.T. Kurland, Seizures after head trauma: a population study, Neurology 30 (1980), 683-689.

[4] J.F. Annegers, J.D. Grabow, L.T. Kurland and E.R. Laws, Jr., The incidence, causes, and secular trends of head trauma in Olmsted County, Minnesota, 1935-1974, Neurology 30 (1980), 912-919.

[5] J.F. Annegers, W.A. Hauser, S.P. Coan and W.A. Rocca, A population-based study of seizures after traumatic brain injuries, New England Journal of Medicine 338 (1998), 20-24.

[6] I. Baguley, S. Slewa-Younan, R. Lazarus and A. Green, Longterm mortality trends in patients with traumatic brain injury, Brain Injury 14 (2000), 505-512.

[7] J.F. Kraus, M.A. Black, N. Hessol, P. Ley, W. Rokaw, C. Sullivan, S. Bowers, S. Knowlton and L. Marshall, The incidence of acute brain injury and serious impairment in a defined population, American Journal of Epidemiology 119 (1984), 186-201.

[8] F. Masson, M. Thicoipe, P. Aye, T. Mokni, P. Senjean, V. Schmitt, P. H. Dessalles, M. Cazaugade, P. Labadens and S. Aquitaine Group for Severe Brain Injuries, Epidemiology of severe brain injuries: a prospective population-based study, Journal of Trauma-Injury Infection \& Critical Care 51 (2001), 481-489.

[9] F. Masson, J. Vecsey, L.R. Salmi, J.F. Dartigues, P. Erny and P. Maurette, Disability and handicap 5 years after a head injury: a population-based study, Journal of Clinical Epidemiology 50 (1997), 595-601.

[10] W. Max, E.J. MacKenzie and D.P. Rice, Head injuries: costs and consequences, J Head Trauma Rehabil 6 (1991), 76-91.

[11] L.J. McGarry, D. Thompson, F.H. Millham, L. Cowell, P.J. Snyder, W.R. Lenderking and M.C. Weinstein, Outcomes and costs of acute treatment of traumatic brain injury, Journal of Trauma-Injury Infection \& Critical Care 53 (2002), 11521159.

[12] L.J. Melton, 3rd, History of the Rochester Epidemiology Project, Mayo Clinic Proceedings 71 (1996), 266-274.

[13] P.N. Nemetz, C. Leibson, J.M. Naessens, M. Beard, E. Kokmen, J.F. Annegers and L.T. Kurland, Traumatic brain injury and time to onset of Alzheimer's disease: a population-based study, American Journal of Epidemiology 149 (1999), 32-40.

[14] B.L. Rish, J.D. Dillon and G.H. Weiss, Mortality following penetrating craniocerebral injuries. An analysis of the deaths in the Vietnam Head Injury Registry population, Journal of Neurosurgery 59 (1983), 775-780.

[15] R.M. Shavelle, D. Strauss, J. Whyte, S.M. Day and Y.L. Yu, Long-term causes of death after traumatic brain injury, American Journal of Physical Medicine \& Rehabilitation 80 (2001), 510-516; quiz 517-519.

[16] D.M. Sosin, J.E. Sniezek and D.J. Thurman, Incidence of mild and moderate brain injury in the United States, 1991, Brain Injury 10 (1996), 47-54.

[17] D.J. Strauss, S. Ashwal, S.M. Day and R.M. Shavelle, Life expectancy of children in vegetative and minimally conscious states, Pediatric Neurology 23 (2000), 312-319.

[18] D.J. Strauss, R.M. Shavelle and T.W. Anderson, Life expectancy of children with cerebral palsy. [comment], Pediatric Neurology 18 (1998), 143-149.

[19] D.J. Strauss, R.M. Shavelle and T.W. Anderson, Long-term survival of children and adolescents after traumatic brain injury, Archives of Physical Medicine \& Rehabilitation 79 (1998), 1095-1100.

[20] D. Thurman, Traumatic brain injury in the United States: A report to Congress, Centers for Disease Control and Prevention, 1999.

[21] D. Thurman and J. Guerrero, Trends in hospitalization associated with traumatic brain injury. [comment], Jama 282 (1999), 954-957.

[22] A. Vazquez-Barquero, J.L. Vazquez-Barquero, O. Austin, J. Pascual, L. Gaite and S. Herrera, The epidemiology of head injury in Cantabria, European Journal of Epidemiology 8 (1992), 832-837.

[23] A.E. Walker, H.K. Leuchs, H. Lechtape-Gruter, W.F. Caveness and C. Kretschman, Life expectancy of head injured men with and without epilepsy, Archives of Neurology 24 (1971), 95100.

[24] G.H. Weiss, W.F. Caveness, H. Einsiedel-Lechtape and M.L. McNeel, Life expectancy and causes of death in a group of head-injured veterans of World War I, Archives of Neurology 39 (1982), 741-743. 